\title{
Operated low grade astrocytomas: a long term PET study on the effect of radiotherapy
}

U Roelcke, K von Ammon, O Hausmann, D L Kaech, W Vanloffeld, H Landolt, J A Rem, O Gratzl, E W Radü, K L Leenders

\begin{abstract}
The role of postoperative radiotherapy in patients with low grade gliomas is not established yet. PET with ${ }^{11} \mathrm{C}$ methionine (MET) and ${ }^{18}$ F fluorodeoxyglucose (FDG) was used to perform cross sectional comparisons as well as within patient follow up studies in 30 operated patients with fibrillary astrocytoma WHO II. Uptake of tracer by tumour was quantified by radioactivity concentration ratios in tumour over contralateral brain (T/C). Comparing patients who did $(n=13)$ or did not $(n=17)$ receive external radiotherapy subsequent to first tumour resection, no differences in MET and FDG T/C between both groups were found during a postoperative period of 94 months (when recurrence and malignant progression of low grade astrocytomas are expected). Malignant progression occurred at a similar rate in both patient groups at a mean (SD) postoperative interval of 46 (26) months. Irrespective of whether radiotherapy was applied or not, malignant tumour recurrences showed higher T/C values (MET: 1.70 (0.64), FDG: $0.98(0.23)$ ) than recurrences without signs of malignancy (MET: $1.21(0.21)$, FDG: 0.82 (0.08)) (MannWhitney: MET $p=0.086$, FDG $p=0.035$ ). The data show a relative lack of radiotherapy administered immediately after first tumour resection. In the course of disease, patients with tumours undergoing malignant progression may be identified with PET tracer methods.

(F Neurol Neurosurg Psychiatry 1999;66:644-647)
\end{abstract}

Keywords: low grade astrocytoma; radiotherapy; positron emission tomography

Cerebral low grade gliomas (LGGs) account for $30 \%$ to $40 \%$ of all gliomas. After operation, patients with LGGs may live several years relatively symptom free with an average interval of 2 to 4 years until tumour recurrence. However, up to $50 \%$ of patients deterioriate more rapidly due to tumour progression towards higher malignancy, which may drastically reduce the quality and duration of life. ${ }^{1}$

Different policies are being pursued in the management of suspected LGG: biopsy or resection with or without subsequent radiotherapy, or delayed resection plus radiotherapy of suspected LGG. ${ }^{1}$ In particular, the timing of radiotherapy remains a matter of debate. Although its efficacy has not been established in prospective studies, a possible rationale for postoperative radiotherapy is provided by the finding of anaplastic tumour cell foci, which may microscopically already be present in low grade astrocytomas. It was assumed that destruction of these foci by radiotherapy could prevent malignant progression, and could delay tumour recurrence, particularly in patients with partial tumour resection. ${ }^{2}$

In the present study, we used PET and the tracers ${ }^{11} \mathrm{C}$ methionine (MET) and ${ }^{18} \mathrm{~F}$ fluorodeoxyglucose (FDG) to consider the issue of external radiotherapy from an in vivo tumour biochemical point of view. PET studies have shown that uptake of tracer by the tumour shows high correlation with brain tumour malignancy and prognosis. ${ }^{3}$ Regarding the above mentioned considerations about malignant progression of operated patients with LGG, we tested whether tracer uptake by the tumour is higher in non-irradiated than in irradiated patients during a period when recurrence and malignant progression can be expected. ${ }^{1}$ This would strongly support radiotherapy efficacy against development of malignant progression.

\section{Patients and methods}

PATIENT SELECTION

We studied 30 operated patients with supratentorial, unilateral fibrillary astrocytomas WHO II, who did (group A, n=13) or did not (group $\mathrm{B}, \mathrm{n}=17$ ) receive radiotherapy subsequent to first operation. Patients of both groups were well controlled for age (mean (SD) A: 39 (14), B: 34 (9) years,) and for the interval between operation \pm radiotherapy and the time of the PET studies (A: 43 (31), B: 40 (26) months, range 7 to 94 ). The proportion of gross total resection was similar in group A $(39 \%)$ and B $(47 \%)$. Out of group $A$ and $B$, four and six patients respectively participated in follow up PET studies which were scheduled at 1 and 2 years after their baseline study. For these patients, PET studies were performed at the following postoperative intervals ( \pm radiotherapy): baseline: 29 (13) (A), 26 (15) (B) 
Tumour tracer uptake

\begin{tabular}{|c|c|c|}
\hline & \multicolumn{2}{|l|}{$T / C$ ratio } \\
\hline & $M E T$ & $F D G$ \\
\hline \multicolumn{3}{|l|}{ Operated tumours: } \\
\hline All RT+ $(n=13)$ & $1.31(0.42)$ & $0.90(0.16)$ \\
\hline All RT $-(\mathrm{n}=17)$ & $1.33(0.40)$ & $0.82(0.10)$ \\
\hline \multicolumn{3}{|l|}{ Malignant progression: } \\
\hline Yes $(n=6)$ & $1.70(0.64) \mathrm{a}$ & $0.98(0.23) b$ \\
\hline No $(n=24)$ & $1.21(0.21)$ & $0.82(0.08)$ \\
\hline \multicolumn{3}{|c|}{ Tumours at the time of first presentation: $\dagger$} \\
\hline Fi AC WHO II $(n=7)$ & $1.38(0.45) \mathrm{c}$ & $0.76(0.17) \mathrm{d}$ \\
\hline Glioblastoma $(n=13)$ & $2.57(0.88)$ & $1.25(0.32)$ \\
\hline
\end{tabular}

$\mathrm{T} / \mathrm{C}$ ratio=ratio of activity concentrations in tumour $(\mathrm{T})$ over contralateral $(\mathrm{C})$ brain. ${ }^{\star}$ Data of all operated patients are split by the presence/absence of malignant progression. †Patients are reported on elsewhere. ${ }^{4}$ FiAC $=$ fibrillary astrocytoma WHO II. Mann-Whitney $\mathrm{p}$ : $a=0.086$; $\mathrm{b}=0.035 ; \mathrm{c}=0.020 ; \mathrm{d}=0.004$. months; first follow up: 43 (11) (A, n=4), 40 (14) $(B, n=6)$ months; second follow up: 54 (14) $(\mathrm{A}, \mathrm{n}=3), 54(16)(\mathrm{B}, \mathrm{n}=3)$ months. Taken together, 46 studies were conducted in 30 patients. Magnetic resonance tomography images were available for all patients. At the time of the PET studies, anticonvulsant drugs were given to seven and 11 patients of group $\mathrm{A}$ and $\mathrm{B}$ respectively, one patient of group A received oral dexamethasone.

The indication for radiotherapy did not depend on the clinical status or neuroradiological or histological findings, but was simply defined by the practice of the referring hospital. Radiotherapy was initiated between 3 and 5 weeks after operation. A total of 56 Gy was given in 28 fractions over 6 to 8 weeks, and was administered as wedge or opponent field irradiation.
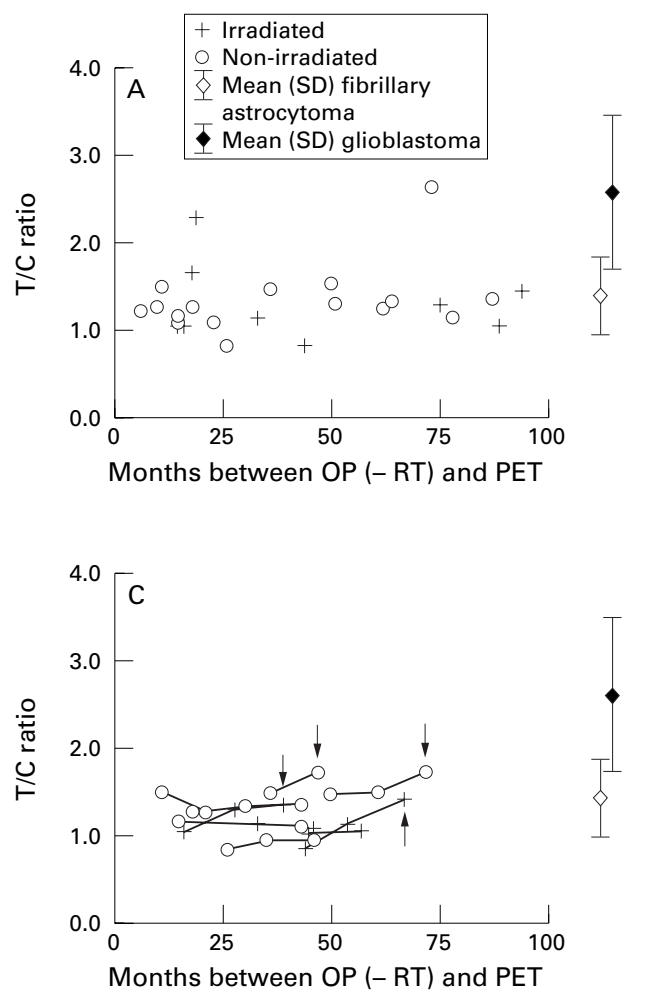

For comparison, we included results of nonoperated patients with fibrillary astrocytoma WHO II ( $n=7$, age 37 (SD 6) years) and glioblastoma ( $\mathrm{n}=8,50$ (SD 13) years) studied immediately before first tumour operation. ${ }^{4}$ The study protocol was approved by the ethics committees of the University Hospitals of Zürich and Basel.

SCANNING PROCEDURE

After bolus injection of the tracers, dynamic PET was acquired on a CTI tomograph (933/ 04-16) (MET: 35 minutes, FDG: 48 minutes after tracer injection). ${ }^{4} \mathrm{MET}$ and FDG were performed at the same day; the interval between both studies was 3 to 4 hours.

\section{DATA ANALYSIS}

Because MET uptake gives a good tumour delineation in low grade gliomas, ${ }^{4}$ elliptical tumour regions of interest (ROIs) were first taken from the MET studies. ROIs were placed at the most active immediate border zone of the surgical defect, as tumours are known to regrow in this area. ROIs of the MET scans were then copied on the corresponding planes of the FDG scans. In five studies in which MET scans were not available, the same approach was used directly in the FDG studies. ROIs from the not affected side of the brain were taken from the whole hemisphere contralateral to the tumour. Uptake of tracer by the tumour was quantified by calculating ratios of activity concentrations (counts/ml) in tumour
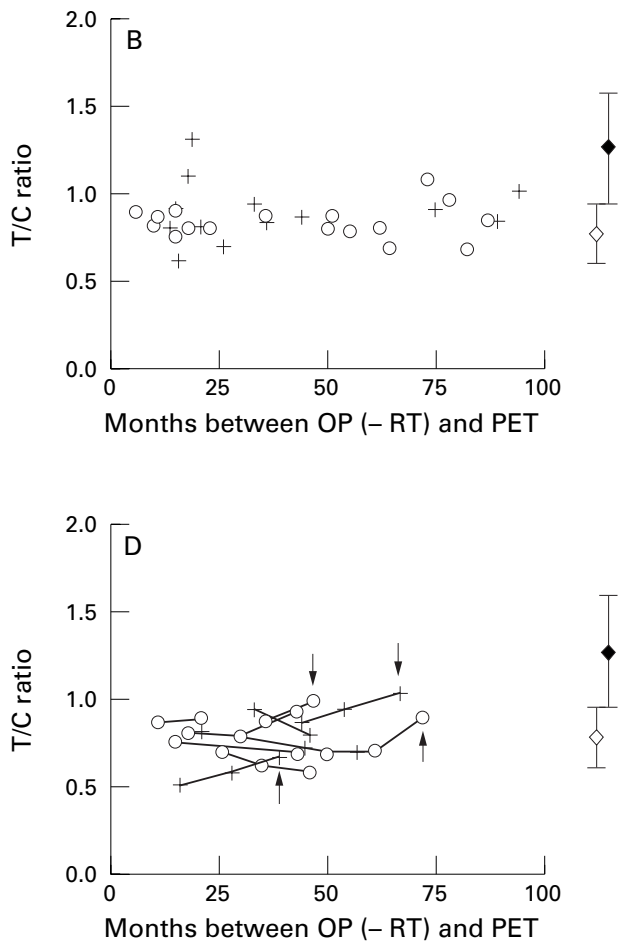

Relation between tumour tracer uptake and the interval between first operation \pm radiotherapy, and PET study. Crosses=irradiated (group $A$ ), open circles=non-irradiated $(B)$ patients with residual tumour or recurrence of fibrillary astrocytoma. Right side plot: mean (SD) of fibrillary astrocytoma (open diamond) and glioblastoma (filled diamond) studied before first operation. ${ }^{4}(A, B)$ Comparison between patients of group $A$ and $B .(C, D)$ Follow up within patients. Patients with malignant progression at the time of reoperation are identified with arrows (all PET studies were performed before reoperation). 
(T) over contralateral (C) brain 35 (MET) and 48 (FDG) minutes after tracer injection. ${ }^{4}$

STATISTICS

Between group comparisons were performed using the Mann-Whitney test.

\section{Results}

Irrespective of whether radiotherapy was administered (group A) or not (group B), operated patients with fibrillary astrocytoma WHO II showed MET and FDG T/C values in the range of non-operated patients with WHO II studied at the time of first tumour presentation (table). The figure (A, B) shows the relation between uptake of tracer by the tumour and the time interval (6 to 94 months) between operation \pm radiotherapy and PET study for all patients of groups A and B. The $\mathrm{T} / \mathrm{C}$ ratios showed no differences between these groups. Similar results were obtained for the series of intraindividual follow up investigations (11 to 72 months; fig C, D). The figure (C, D) also shows increasing $\mathrm{T} / \mathrm{C}$ values with time from first operation in patients in whom malignant progression was verified at the time of reoperation (see below).

At the time of the PET study (for those patients who were serially studied: last follow up study), 3/13 (23\%) of group A patients, and $3 / 17(18 \%)$ of group B patients showed MRI signs of malignant progression (marked contrast enhancement, peritumorous oedema). This was histologically confirmed at reoperation in two patients of each group. The interval between operation \pm radiotherapy and malignant progression was 46 (SD 26) months. Splitting data of all group A and B patients only by the presence or absence of malignant progression yielded substantially higherT/C values in patients with malignant progression (table). Patients exhibiting malignant progression were on average older (40 (13) years) than patients without malignant progression (33 (11) years) (Mann-Whitney $\mathrm{p}=0.082)$.

\section{Discussion}

This PET study considered the effect of external beam radiotherapy on tracer uptake by the tumour. Using this as biochemical indicator of tumour dignity, we found similar uptake values in both irradiated and non-irradiated patients with fibrillary astrocytoma (WHO II) during a period in which recurrences usually occur, and when a considerable percentage of tumours previously diagnosed as WHO II is expected to progress towards higher malignancy. ${ }^{1}$ Uptake of tracer by the tumour in our patients was also similar in patients who underwent either gross total or partial resection. Thus our data suggest that the rate of malignant progression could occur independently from residual tumour size and radiotherapy. In the early series of incompletely resected low grade gliomas of various subtypes reported by Leibel et $a l^{5}$ there was a survival advantage of the radiotherapy over the non-radiotherapy group. On the other hand, particularly considering the relation between residual tumour size and survival, Miralbell et al more recently noted that the histological subtype rather than tumour size correlates with survival. ${ }^{6}$ Also the results of a multivariate analysis published by Shibamoto et al argue against a prognostic influence of residual tumour size. ${ }^{7}$ Assuming that histological subtypes determine the overall biological behaviour, which in turn is reflected in alterations of tumour cell metabolism, ${ }^{3}$ our findings on malignant tumour progression suggest that in vivo measurement of biochemical indicators could provide a direct link to the management of operated patients with LGG.

The immediate effect of interstitial radiotherapy on brain tumour metabolism was studied by Würker et al in 10 patients with LGG (WHO I, II) before and 1 year after stereotactic implantation of ${ }^{125} \mathrm{I}$ seeds. ${ }^{8}$ With FDG, increases or decreases of $\mathrm{T} / \mathrm{C}$ values were noted, whereas with MET, a dose dependent decrease to more than $40 \%$ was found. Wang et al reported that postoperative external beam radiotherapy induced reductions of tumour FDG uptake in various primary and secondary brain tumours. ${ }^{9}$ However, the investigation of immediate effects of postoperative external radiotherapy may be hampered by the fact that within weeks to months from resection, disruption of the blood-brain barrier as well as altered tissue biochemistry as a direct consequence of tumour resection may confound the interpretation of tumour tracer uptake. ${ }^{10}$ Therefore, the relevance of these findings in terms of the biological behaviour of irradiated LGG remains open.

Several clinical studies have considered the timing of any intervention during the course of patients with LGG. In the series of Recht et al data from 26 patients suspected to have LGG, in whom immediate therapy was withheld until deemed necessary, were compared with the data from 20 patients with LGG treated immediately after diagnosis. ${ }^{11}$ The rate of malignant progression, as well as the time elapsed from initial diagnosis until malignant progression, was similar in both groups. In the study of Leighton et al, the timing of radiotherapy was not a significant prognostic variable. ${ }^{12}$ In addition, delaying radiotherapy in a small cohort of patients with LGG produced a similar percentage of 10 year survival as in patients who received radiotherapy immediately after tumour resection. ${ }^{13}$ As our PET data show a relative lack of effect of early radiotherapy, they seem to be in accordance with these clinical data. As it can be expected that metabolic tumour derangement due to malignant progression precedes changes in tumour morphology and size as shown by MRI, PET tracer methods may identify subjects undergoing malignant progression. It remains to be determined whether these patients may then benefit from radiotherapy.

This study was supported by grants from the Swiss Cancer League (FOR 491, 452.90).

1 Morantz RA. The management of the patient with a low-grade cerebral astrocytoma. In: Morantz RA, Walsh Marcel Dekker, 1994:387-415. 
2 Soffietti R, Chio A, Giordana MT, et al. Prognostic factors in well differentiated cerebral astrocytomas in the adult. in well differentiated cereb

3 Di Chiro G. Positron emission tomography using [18F]fluoro-deoxyglucose in brain tumours. A powerful diagno tic and prognostic tool. Invest Radiol 1986;22:360-71.

4 Roelcke U, Radü EW, von Ammon K, et al. Alteration of blood-brain barrier in human brain tumours: comparison of $[18 \mathrm{~F}]$-fluorodeoxyglucose, [11C]-methionine and [82rubidium] using PET. $\mathcal{F}$ Neurol Sci 1995;132:20-7.

5 Leibel SA, Sheline GE, Wara WM, et al. The role of radiation therapy in the treatment of astrocytomas. Cancer 1975;35:1551-7.

6 Miralbell R, Balart J, Matias Guiu X, et al. Radiotherapy for supratentorial low-grade gliomas: results and prognostic factors with special focus on tumour volume parameters. Radiother Oncol 1993;27:112-6.

7 Shibamoto Y, Kitakabu Y, Takahashi M, et al. Supratentorial low-grade astrocytoma. Correlation of computed tomography findings with effect of radiation therapy and prognostic variables. Cancer 1993;72:190-5.
8 Würker M, Herholz K, Voges J, et al. Glucose consumption and methionine uptake in low-grade gliomas after iodine125 brachytherapy. Eur f Nucl Med 1996;23:583-6.

9 Wang GJ, Volkow ND, Lau YH, et al. Glucose metabolic changes in nontumoral brain tissue of patients with brain tumour following radiotherapy. 7 Comput Assist Tomogr 1996;20:709-14.

10 Forsting $M$, Albert FK, Kunze $S$, et al. Extirpation of glioblastomas: MR and CT follow-up of residual tumour and regrowth patterns. Am $\mathcal{F}$ Neuroradiol 1993;14:77-87.

11 Recht LD, Lew R, Smith TW. Suspected low-grade glioma: is deferring treatment safe? Ann Neurol 1992;31:431-6.

12 Leighton C, Fisher B, Baumann G, et al. Supratentorial low-grade glioma in adults: An analysis of prognostic factors and timing of radiation. F Clin Oncol 1997:15:1294301.

13 Knisely JP, Haffty BG, Christopher SR. Early v delayed radiotherapy in a small cohort of patients with supratentorial low grade glioma. $f$ Neurooncol 1997;34:23-9. 